\title{
Interspecific Competition for Colonization of Maize Plants Between Fusarium proliferatum and Fusarium verticillioides
}

\author{
A. Reyes Gaige, T. Todd, and J. P. Stack ${ }^{\dagger}$ \\ Department of Plant Pathology, Kansas State University, Manhattan, KS 66506, U.S.A.
}

\begin{abstract}
Fusarium proliferatum and $F$. verticillioides are mycotoxin-producing, seedborne pathogens of maize. They are often asymptomatic in seed, eluding symptom-based detection. Experiments were conducted in nonsterile soil to determine whether interspecific competition influenced establishment in maize plants of an introduced isolate of $F$. proliferatum or $F$. verticillioides. Hygromycin-resistant, green fluorescent protein (GFP) transformed (GFP-tagged) $F$. proliferatum ( $F$. proliferatum-green) and hygromycin-resistant, monomeric red fluorescent protein (mRFP) transformed (mRFP-tagged) $F$. verticillioides ( $F$. verticillioides-red) strains were developed to provide molecular markers to track fungal establishment. Heat-killed Fusarium-free maize seed, colonized with $F$. proliferatum-green or $F$. verticillioides-red by immersion in a spore suspension for $16 \mathrm{~h}$, served as the source of inoculum. The ability of $F$. proliferatum-

consecutive root segments and three consecutive stem segments. A TaqMan multiplex real-time quantitative PCR protocol was developed to identify and quantify $F$. proliferatum-green and $F$. verticillioides-red from each plant segment from each treatment; the experiment was repeated three times. This experiment confirmed that $F$. proliferatum-green and $F$. verticillioides-red effectively colonized roots and stems of the maize plant already colonized with the other species. Prior colonization of maize tissues by $F$. verticillioides-red $(P=0.6749)$ and other seedborne microorganisms $(P=0.1910)$ reduced but did not prevent subsequent colonization by $F$. proliferatum-green. Similarly, prior colonization of maize tissues by $F$. proliferatum-green $(P=0.7032)$ and other seedborne microorganisms $(P=0.1447)$ reduced but did not prevent subsequent colonization by $F$. verticillioides-red.
\end{abstract} green and $F$. verticillioides-red to colonize viable maize plants already colonized by the other species was determined. Maize plants were retrieved from soil after 14 days and DNA was extracted from three
Keywords: Fusarium, interspecific competition, invasion, maize, real time qPCR
Fusarium proliferatum and F. verticillioides belong to the Fusarium fujikuroi species complex (FFSC) (O'Donnell et al. 1998a, b). These two fungal species share many morphological and biochemical characteristics and misidentification can occur (Leslie and Summerell 2006). They have the ability to produce fumonisins and other mycotoxins in contaminated grain (Leslie and Summerell 2006). Fumonisin B1 is toxic to humans and other animals due to inhibition of sphingolipid metabolism and cell cycle regulation (Riley et al. 1996). It has been associated with esophageal cancer, liver cancer, and neural tube defects (Desjardins 2006; Marasas 1995; Marasas et al. 2004; Thiel et al. 1992). Some strains of $F$. proliferatum are reported to produce FB1 in culture at more than $6,000 \mu \mathrm{g} / \mathrm{g}$ (Leslie et al. 2004). Fumonisin contamination has been associated with $F$. proliferatum infection of maize, animal feeds, and other agricultural commodities (Leslie et al. 2004; Logrieco et al. 1998; Pascale et al. 2002). In horses, it causes leukoencephalomalacia and, in swine, it causes pulmonary edema (Ross et al. 1990). In addition, they are both seedborne in maize and important colonizers of maize plants (Logrieco et al. 2002; Munkvold 2003). Moreover, seed can vector exotic pathogens into new locations (Elmer 2001), and a previous study showed that $F$. proliferatum VCG US5 was likely introduced to Australia from the United States by importation of asparagus seed or

${ }^{\dagger}$ Corresponding author: J. P. Stack; jstack@ksu.edu

Funding: Support for this research was provided by the National Agricultural Biosecurity Center and the European Union 7th Framework Plant and Food Security (project number 261752) and the Kansas Agricultural Experiment Station (contribution number 16-174-J).

*The $\boldsymbol{e}$-Xtra logo stands for "electronic extra" and indicates that two supplementary figures and one supplementary table are published online.

The author(s) declare no conflict of interest.

Accepted for publication 28 February 2020.

(C) 2020 The American Phytopathological Society crowns (Elmer et al. 1999). The introduction of maize seed colonized by $F$. proliferatum or $F$. verticillioides isolates may result in the introduction and establishment of undesirable isolates into new areas.

To colonize maize plants, these Fusarium spp. compete against each other, as well as with other microorganisms, including other fungi and bacteria. Competition for a niche in maize plants comes from other seedborne microorganisms, as well as from microorganisms outside the seed (Marín et al. 1998a, b, c). These past investigations suggested that strains of $F$. proliferatum and $F$. moniliforme (FFSC species) were very competitive against a range of other maize colonizers. Niche overlap indices, based on patterns of carbon or nitrogen sources, found that $F$. proliferatum was more competitive than $F$. moniliforme (FFSC species) and that, depending on abiotic factors (water potential and temperature), different niches were occupied by other species (Marín et al. 1998a, b). In addition, it was found that the fungal interspecific interactions resulted in stimulation of fumonisin production by $F$. proliferatum and $F$. moniliforme (FFSC species) (Marín et al. 1998c). These past studies were all done in vitro on culture media or on seed in chambers. Extrapolation of activity and competitive ability in natural environments (e.g., soils in agroecosystems) is not possible, though essential for estimating the significance to invasion potential. For this investigation, invasion potential can be defined as the ability of an organism to arrive or be introduced, spread, and become established in a new environment having a negative effect in the resident organism (Desprez-Loustau et al. 2007).

Furthermore, abiotic conditions can enhance or diminish the invasion potential of these organisms. Previous studies in synthetic media demonstrated the importance of water activity, $\mathrm{pH}$, and temperature for the growth of $F$. proliferatum and other Fusarium spp. and fungi (Marín et al. 1995, 1998b). The influence of abiotic factors on fungal interactions has been extensively demonstrated in a range of ecosystems for different species (Magan and Lacey 1984, 1985; Ramakrishna et al. 1993). Fusarium spp. from the FFSC ( $F$. proliferatum and $F$. verticillioides) have similar abiotic requirements (Leslie and Summerell 2006), which makes them ideal for interspecific competition experiments.

Considering the ecological similarities of $F$. proliferatum and $F$. verticillioides, it is likely that they also have similarities in their life cycles. In the colonization of maize plants, we hypothesize that these 
Fusarium spp. compete against each other for the same resources and that colonization success is dependent on where the inoculum is located (seedborne to the growing plant or external to the growing plant). In this study, we investigated the competitive ability of $F$. proliferatum with $F$. verticillioides and other naturally occurring seedborne microorganisms for a niche in maize plants. To facilitate strain discrimination of the two Fusarium spp., they were transformed using Agrobacterium tumefaciens-mediated transformation; the $F$. proliferatum strain was transformed to express a green fluorescent protein (GFP-tagged) and hygromycin resistance ( $F$. proliferatum-green), and the $F$. verticillioides strain was transformed to express a monomeric red fluorescent protein (mRFP-tagged) and hygromycin resistance $(F$. verticillioidesred). We developed a multiplex TaqMan real-time quantitative PCR (qPCR) assay with high specificity and sensitivity for the detection and quantification in plants of $F$. proliferatum-green and $F$. verticillioides-red using their respective fluorescence genes as targets.

Preliminary evidence demonstrated that $F$. proliferatum-green can grow out of a source of inoculum and actively disperse by hyphal growth at least $1.5 \mathrm{~cm}$ in nonsterile soil (Reyes Gaige et al. 2019). The purpose of this investigation was to complement those findings by studying the potential establishment of $F$. proliferatum-green and $F$. verticillioides-red. In this investigation, potential establishment can be defined as the ability of an exogenous organism to grow out of a source of inoculum and colonize plant material. This was done by assessing their competitive ability against the other species and other seedborne microorganisms. The objectives of this investigation were to (i) design specific and sensitive primers and probe sets to identify $F$. proliferatum-green and $F$. verticillioides-red in multiplex real-time qPCR assays and (ii) use the real-time qPCR data to quantify the amount of $F$. proliferatum-green and $F$. verticillioides-red in maize plant in the different treatments tested. The hypotheses for this investigation were (i) colonization success of the maize plant by Fusarium spp. is dependent on the location of the inoculum, (ii) roots and stems will have similar levels of colonization, and (iii) colonization of roots and stems will be greater for segments that are closer to the seed. Several investigations have studied infection of maize by different Fusarium spp. (Chulze et al. 2000; Cotten and Munkvold 1998; Iglesias et al. 2010; Leslie et al. 1990; Logrieco and Bottalico 1988; Logrieco et al. 2002; Munkvold 2003; Stępień et al. 2011); however, information about two-species infection and colonization of maize at the same time from either seedborne or soilborne inoculum sources (or both) is lacking. Therefore, the results of this investigation will increase understanding of the invasion potential of $F$. proliferatum and $F$. verticillioides in maize.

\section{Materials and Methods}

Fusarium isolates. $F$. proliferatum and $F$. verticillioides were isolated from asymptomatic maize seed (DuPont Pioneer hybrids 32 N70 and 33B54). Seed were surface sterilized with a 10\% sodium hypochlorite solution for $1 \mathrm{~min}$, rinsed in distilled water for $30 \mathrm{~s}$, and plated onto Nash-Snyder (NS) medium (Leslie and Summerell 2006). Isolates were single spored and grown on NS medium at $27^{\circ} \mathrm{C}$ for 7 days, and identified as $F$. proliferatum or $F$. verticillioides by morphological and molecular characteristics, including amplification and sequencing of the TEF- $1 \alpha$ and $F U M$ genes (O'Donnell et al. 1998b; Stępień et al. 2011). One isolate (Fp-70-2-5) confirmed as $F$. proliferatum and another isolate $(\mathrm{Fv}-54-3-5)$ confirmed as $F$. verticillioides were used in this study. Other Fusarium spp. isolated from maize seed included $F$. subglutinans, $F$. thapsinum, and $F$. andiyazi (J. Stack, unpublished data).

A. tumefaciens-mediated transformation of isolates and characterization of transformants. A plasmid (pBV126, provided by Dr. Barbara Valent) carrying genes encoding GFP and hygromycin resistance was used for the transformation of $F$. proliferatum. A plasmid (pBV216, provided by Dr. Barbara Valent) carrying genes encoding $\mathrm{mRFP}$ and hygromycin resistance was used for the transformation of $F$. verticillioides. Transformations followed published protocols (Mullins et al. 2001) and were considered successful when $F$. proliferatum expressed the GFP and hygromycin resistance and $F$. verticillioides expressed the $\mathrm{mRFP}$ and hygromycin resistance.
Transformed strains were tested to confirm that the insertions were mitotically stable by multiple, sequential subculturing followed by comparison with the parent isolates, Fp-70-2-5 and Fv-54-3-5. Characterization of transformed strains included morphology, hyphal growth rate, pathogenicity in apple fruit, colonization of maize seed, and expression of both the green fluorescence trait and hygromycin resistance, as previously described (Reyes Gaige and Stack 2015; Reyes Gaige et al. 2019).

Southern blot analysis. The number of insertions of the fluorescent genes in the transformants was determined by Southern blot analysis of genomic DNA of the transformed strains. For each reaction, $5 \mu \mathrm{g}$ of genomic DNA was digested with EcoRI-HF (New England Biolabs, Ipswich, MA, U.S.A.) overnight, separated in a $0.7 \%$ agarose gel, and blotted to a positively charged nylon membrane using an upward alkaline capillary transfer. Probe preparation and labeling, hybridization of the probe, and washes of the nylon membrane were done using the AlkPhos Direct Labeling and Detection System with CDP-Star (GE Healthcare Life Sciences, Pittsburg, PA, U.S.A.). A 557-bp-long gene fragment was selected as the probe for the green fluorescence gene and a 647 bp-long gene fragment was selected as the probe for the red fluorescence gene to detect the gene copy number. The two probes were hybridized onto the membrane at the same time for their detection (Supplementary Fig. S1). Probes were synthesized as gBlocks gene fragments (Integrated DNA Technologies, Inc., Coralville, IA, U.S.A.) (Supplementary Table S1).

Source of inoculum and bait plants generation. Maize seed were heat killed and disinfected in water at $75^{\circ} \mathrm{C}$ for $20 \mathrm{~min}$. The heat-killed seed were plated in NS medium and no Fusarium growth was observed in the plate after 7 days. These nonviable, Fusariumfree (seedborne organisms were killed by the high temperature of water bath) seed were submersed in spore suspensions $\left(1 \times 10^{6}\right.$ spores/ $\mathrm{ml})$ and placed on the rotary shaker $(80 \mathrm{rpm})$ at $25^{\circ} \mathrm{C}$ for $16 \mathrm{~h}$ to allow colonization by $F$. proliferatum ( $F$. proliferatum-green, hygromycin resistant, green fluorescent), or $F$. verticillioides ( $F$. verticillioidesred, hygromycin resistant, red fluorescent). Spore suspensions were prepared by using sterile loops to collect respective Fusarium spores growing in Spezieller Nahrstoffarmer agar and placing them in autoclaved distilled water; a hemocytometer was used to obtain desired concentrations. Nonviable, Fusarium-free seed placed in sterile water served as the negative control. Colonized seed were surface sterilized (10\% sodium hypochlorite for $1 \mathrm{~min})$ and rinsed in distilled water (30 s); they served as the sources of inoculum.

Noncolonized and naturally or artificially colonized viable seed were surface sterilized (10\% sodium hypochlorite), rinsed with distilled water ( $30 \mathrm{~s})$, and used as bait plants. To artificially colonize viable maize seed, we modified a published protocol to eradicate and cure maize seed (Daniels 1983) of naturally occurring Fusarium spp. by exposing the seed to a $65^{\circ} \mathrm{C}$ water bath for $4 \mathrm{~min}$, then recolonized the seed by placing them in spore suspensions as described above. After colonization, these viable seed were surface sterilized with $10 \%$ sodium hypochlorite for $1 \mathrm{~min}$ and rinsed in distilled water for $30 \mathrm{~s}$.

Colonization of plants and qPCR data analyses. Colonization of plants was investigated in nine treatments (Table 1) to study $F$. proliferatum-green and $F$. verticillioides-red performance within different competition events. The experiment used a split-plot design, in which each experiment in the growth chamber was treated as the whole plot and each individual treatment within the growth chamber was treated as a subplot. The experiment was conducted three times in the same growth chamber (Conviron PGR15) under the same environmental conditions: $25^{\circ} \mathrm{C}$ in $14 \mathrm{~h}$ of light and $21^{\circ} \mathrm{C}$ in $10 \mathrm{~h}$ of darkness. In the first experiment, 10 replicate plants per treatment were used and, in the second and third experiments, 5 replicate plants per treatment were used. All plants were grown in SC10 Super conetainers with a depth of $21 \mathrm{~cm}$ and a diameter of $3.8 \mathrm{~cm}$ (Stuewe \& Sons, Inc.). Two sources of seed with heat-killed inoculum were placed adjacent to the planted seed for all treatments. In all experiments, we used soil collected from a Kansas State University experiment field in Hutchinson, KS (Reyes Gaige et al. 2019). 
Treatments 1,3 , and 5 were compared to assess the ability of $F$. proliferatum-green to grow from a source of inoculum and colonize maize plants under different competition conditions; maize plants previously colonized with $F$. verticillioides-red (treatment 1 ), maize plants previously colonized with naturally occurring microorganisms (treatment 5), or cured maize plants (treatment 3). Treatments 2, 4, and 6 were compared to assess the ability of $F$. verticillioides-red to grow from a source of inoculum and colonize maize plants under different competition conditions; maize plants previously colonized

Table 1. Competition between Fusarium proliferatum (Fp-G) and F. verticillioides (Fv-R) for colonization of maize seedlings

\begin{tabular}{lll}
\hline Treatments $^{\mathbf{a}}$ & Source of inocula & $\begin{array}{c}\text { Target maize seedlings } \\
\text { (colonized with) }\end{array}$ \\
\hline 1 & Fp-G & Fv-R \\
2 & Fv-R & Fp-G \\
3 & Fp-G & Noncolonized \\
4 & Fv-R & Noncolonized \\
5 & Fp-G & Natural seed microflora \\
6 & Fv-R & Natural seed microflora \\
7 & Noncolonized & Noncolonized \\
8 & Noncolonized & Fv-R \\
9 & Noncolonized & Fp-G \\
\hline
\end{tabular}

a Maize seed were heat cured in treatments 1,2,3,4, 8, and 9 .

${ }^{b}$ For source of inoculum, maize seed were heat killed and then subsequently colonized by Fp-G or Fv-R; noncolonized heat-killed maize seed were used in some control treatments $(7,8$, and 9$)$.

${ }^{c}$ For target seedlings, maize seed were heat cured and colonized by Fp-G or Fv-R (treatments 1, 2, 8, and 9), heat cured and not colonized by Fp-G or Fv$\mathrm{R}$ (treatments 3, 4, and 7), or not heat cured (treatments 5 and 6). with $F$. proliferatum-green (treatment 2 ), maize plants previously colonized with naturally occurring microorganisms (treatment 6), or cured maize plants (treatment 4). Treatment 7, the negative control for each experiment, was included to ensure that no cross-contamination occurred among treatments. Treatment 8 was compared with treatment 1 to assess whether the presence of $F$. proliferatum-green had an effect on $F$. verticillioides-red. Treatment 9 was compared with treatment 2 to assess whether $F$. verticillioides-red had an effect on $F$. proliferatumgreen.

Fourteen days after planting, plants were retrieved from the soil and thoroughly rinsed with water to remove soil and organic matter particles that had adhered to the plant. For each plant, the root and the stem were divided in three equal segments of $3 \mathrm{~cm}$ each. The segments were labeled R1, R2, and R3 for segments collected from the root, R1 being closest to the seed and R3 furthest away, and $\mathrm{S} 1, \mathrm{~S} 2$, and S3, for segments collected from the stem, S1 being closest to the seed and S3 furthest away (Fig. 1).

These segments were sterilized with $10 \%$ sodium hypochlorite for $1 \mathrm{~min}$ and rinsed with water for $1 \mathrm{~min}$. Genomic DNA was extracted from the root and stem segments using the GeneJet Plant Genomic DNA Purification kit (Thermo Fisher Scientific) according to the manufacturer's instructions. DNA extractions were quantified using Qubit fluorometric quantification 3.0 (Thermo Fisher Scientific) and adjusted to $10 \mathrm{ng} / \mu \mathrm{l}$ for analysis of $F$. proliferatum-green and $F$. verticillioides-red in real-time qPCR. The quantification data were logarithmically transformed, and analysis was done using the GLIMMIX procedure (PROC GLIMMIX) in SAS (version 9.3; SAS Institute Inc., Cary, NC, U.S.A.). To analyze the significance of the interactions among variables, three experiments were done composed of nine treatments, two plant parts (roots or stem), and three segments per part (R1, R2, R3, S1, S2, and S3); a Bonferroni
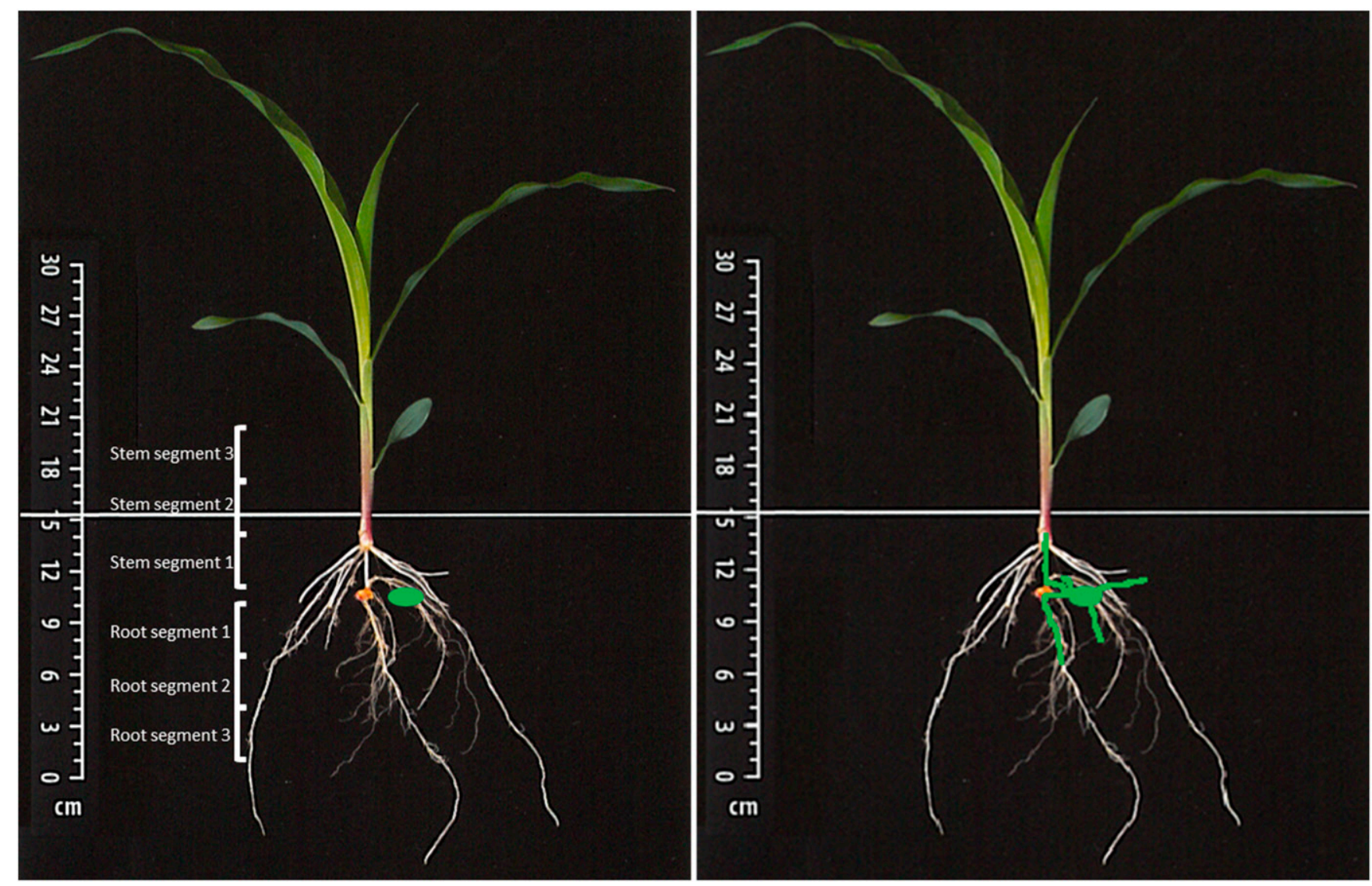

Fig. 1. Graphical representation of experimental set-up for treatment 3, showing the three stem and three root segments sampled for the presence of Fusarium proliferatum (Fp-G) or $F$. verticillioides ( $F v-R$ ). Heat-treated cured seed were planted with nonviable heat-killed seed colonized with $F p-G$ or $F v-R$ (for treatment 3 , it was $F p-G$ ). The heat-killed colonized seed served as the source of inoculum; Fp-G and Fv-R grew through the soil and colonized the bait plants. In some treatments, the bait plants were previously colonized by Fp-G or Fv-R. Graph modified from Abendroth et al. (2011). 
correction of $P$ values was calculated; and the critical $P$ value $(\alpha)$ was divided by the number of comparisons. This step was necessary to account for the multiple comparisons (statistical tests) of data that increased the probability of obtaining a significant result due to chance. The Bonferroni adjustments reduced the chance of obtaining false-positive results (type I errors) (Verhoeven et al. 2005).

Primer and probe design. Sequences of GFP and mRFP genes were used to design two primer and probe sets specific to each gene (Table 2). Instead of relying on microscopy to visualize competition between the transformed strains, we used the inserted GFP or mRFP genes to design markers for their detection and quantification using qPCR. This enabled us to detect only the transformed strains we had inoculated with rather than using species-specific markers that may have detected background strains of $F$. proliferatum or $F$. verticillioides. The two fluorescence genes were aligned using Geneious (version 7.1.7) to find polymorphic regions to ensure specificity in the design of the primer and probe sets, and they were designed using Primer3. Specificity was also confirmed in silico by using BLASTn to screen the primer and probe sequences. Primer thermodynamics, internal structures, and self-dimer formation were examined in silico with mFold (Zuker 2003). Primers and double-quencher probes sets were synthesized by IDT (Integrated DNA Technologies Inc). The probe for the detection of GFP in the transformed $F$. proliferatum was 5'-/6-carboxyfluorescein (6-FAM)/ ZEN/3' Iowa Black FQ, and the probe for the detection of mRFP in the transformed $F$. verticillioides was $5^{\prime}$-/cyanine5 (Cy5)/TAO/ 3' Iowa Black RQ.

Real-time qPCR amplification of treatments. Amplification reactions were carried out in 20- $\mu$ l mixtures containing $10 \mu \mathrm{l}$ of Sso Advanced Universal Probes Supermix (Bio-Rad), $1 \mu 1(5 \mu \mathrm{M})$ of mixture of forward and reverse primer for GFP, $1 \mu \mathrm{l}(5 \mu \mathrm{M})$ of mixture of forward and reverse primer for mRFP, $1 \mu \mathrm{l}(5 \mu \mathrm{M})$ of probe for GFP, $1 \mu \mathrm{l}(5 \mu \mathrm{M})$ of probe for mRFP, $5 \mu l$ of nuclease-free water, and $1 \mu \mathrm{l}$ of genomic DNA. A negative control (nuclease-free water) was used in each run to control for cross-contamination. In addition, for each run, one of the genomic DNA samples was run in three replicates. Cycling parameters included an initial hold for $3 \mathrm{~min}$ at $95^{\circ} \mathrm{C}$, followed by 40 cycles at $95^{\circ} \mathrm{C}$ for $15 \mathrm{~s}$ and $58^{\circ} \mathrm{C}$ for $30 \mathrm{~s}$ The assays were performed in a Bio-Rad CFX96 Real-Time System thermocycler (Bio-Rad), and data analysis was done using Bio-Rad CFX Manager software 2.1 (Bio-Rad).

Real-time qPCR sensitivity and spiked assays. The detection limits and accuracy of detection were tested for the two primer and probe sets. Genomic DNA from $F$. proliferatum-green or $F$. verticillioides-red was diluted 10-fold and tested from $1 \mathrm{ng}$ to $10 \mathrm{fg}$ per mixture. Initial DNA concentrations were determined using Qubit fluorometric quantification 3.0 (Thermo Fisher Scientific). Each reaction was performed in five replicates and the average data were used to build a standard curve with threshold cycle $(\mathrm{Ct})$ in the $y$ axis and the logarithm amount of DNA (in nanograms) in the $\mathrm{x}$ axis, using a linear fit. In addition, spiked assays including known quantities of $F$. proliferatum-green and $F$. verticillioides-red genomic
DNA were performed in triplicate in reactions mixed with root or stem extracts from maize (Arif et al. 2013) and without root or stem extracts from maize to assess reaction efficiency (Supplementary Fig. S2).

\section{Results}

Transformant characterization. One transformed strain of $F$. proliferatum ( $F$. proliferatum-green) and one transformed strain of $F$. verticillioides ( $F$. verticillioides-red) having strong fluorescence and stable hygromycin resistance were selected for further characterization. The analyses were performed in these strains as previously described and demonstrated (Reyes Gaige and Stack 2015; Reyes Gaige et al. 2019) using morphological and pathogenicity traits to compare the transformed strains to the parental types. In all characteristics measured, the transformed strains of both species were stable and indistinguishable from their respective parental types.

Southern blot analysis. End-point PCR using the primers developed for the real-time qPCR confirmed the presence of the respective fluorescence genes: GFP in the $F$. proliferatum strain and mRFP in the $F$. verticillioides strain. For the Southern blot analysis, each transformed strain $(F$. proliferatum-green and $F$. verticillioides-red) was replicated three times to ensure accuracy of the results. It was found that they each contained one copy of the respective fluorescence gene; the parental types (negative control) of both transformed strains did not have the fluorescence gene (Supplementary Fig. S1). This indicated that the $\mathrm{Ct}$ values obtained in the real-time qPCR assays could be directly compared and were not affected by different copy numbers of the respective fluorescence genes.

Sensitivity and specificity assay of primers and probes and spiked assays. Primer and probe sets designed for $F$. proliferatumgreen and $F$. verticillioides-red showed high specificity because the real-time qPCR successfully detected $F$. proliferatum-green or $F$. verticillioides-red without cross-reactions. In the sensitivity assays, both primer and probe sets detected as little as $10 \mathrm{fg}$ of genomic DNA. Spiked assays, which included known amounts of genomic DNA from $F$. proliferatum-green and $F$. verticillioides-red in single reactions, showed high sensitivity (10 fg) and high specificity (no cross-reaction). In addition, the spiked assays that included known amounts of genomic DNA from $F$. proliferatum-green and $F$. verticillioides-red and extracts either from maize roots or maize stems also showed high specificity and no inhibition or reduction in sensitivity caused by inhibitors (Fig. 2). The presence of root or stem extracts had no detectable effect on the real-time qPCR for the detection of $F$. proliferatum-green or $F$. verticillioides-red. The doublequenched probes increased signal detection (assay sensitivity) and decreased background fluorescence. The real-time qPCR results were used to build standard curves for $F$. proliferatum-green and $F$. verticillioides-red $\left(R^{2} \geq 0.98\right)$ for the nonspiked and spiked assays (Fig. 2).

Colonization of plants and analyses from real-time qPCR data. Data from the three experiments were analyzed together because the results indicated that there was no statistically significant interaction between experiment and treatments.

Table 2. Real-time quantitative PCR (qPCR) primer and probe sequences for Fusarium proliferatum (Fp-G) and F. verticillioides (Fv-R) and Southern blot probe sequences for $\mathrm{Fp}-\mathrm{G}$ and $\mathrm{Fv}-\mathrm{R}^{\mathrm{a}}$

Real-time qPCR primers and probes and

Southern blot probes

Fp-G real-time qPCR forward primer

Fp-G real-time qPCR reverse primer

Fv-R real-time qPCR forward primer

Fp-G real-time qPCR probe

Fv-R real-time qPCR probe
Fv-R real-time qPCR reverse primer

Sequences

5'-GAACGGCATCAAGGTGAACT-3'
5'-AGCTCAGGTAGTGGTTGTCG-3'
5'-ATGAGGCTGAAGCTGAAGGA-3'
5'-CTCGTTGTGGGAGGTGATG-3'
5'-/6-carboxyfluorescein (6-FAM)-AACATCGAG/ZEN/GACGGCAGCGTG-
3'-Iowa Black FQ
5'-/cyanine5 (Cy5)-ACGACGCCG/TAO/AGGTCAAGACCAC-3'-Iowa Black
RQ

a Fp-G primers produced a 134-bp amplicon and the Fv-R primers produced a 132-bp amplicon. 
Real-time qPCR data for $F$. proliferatum-green treatments 1, 2, 3, 5 , and 9 were analyzed together, and real-time qPCR data for $F$. verticillioides-red treatments $1,2,4,6$, and 8 were analyzed together. The real-time qPCR data for $F$. proliferatum indicated that colonization of maize plants from sources of inoculum differed from colonization of maize plants from the inoculated seed $(P<0.05)$ (Table 3$)$.
This was shown by the amount of $F$. proliferatum-green biomass detected in the plant tissues among the treatments (values shown in parenthesis in the comparisons). There were no significant differences among treatments 1 (1.28 pg), $3(2.22 \mathrm{pg})$, and $5(0.94 \mathrm{pg})$; however, these three treatments were significantly different from treatments $2(5.39 \mathrm{pg})$ and $9(5.45 \mathrm{pg})(P<0.05)$

\section{A Sensitivity Assay of Fp-G}

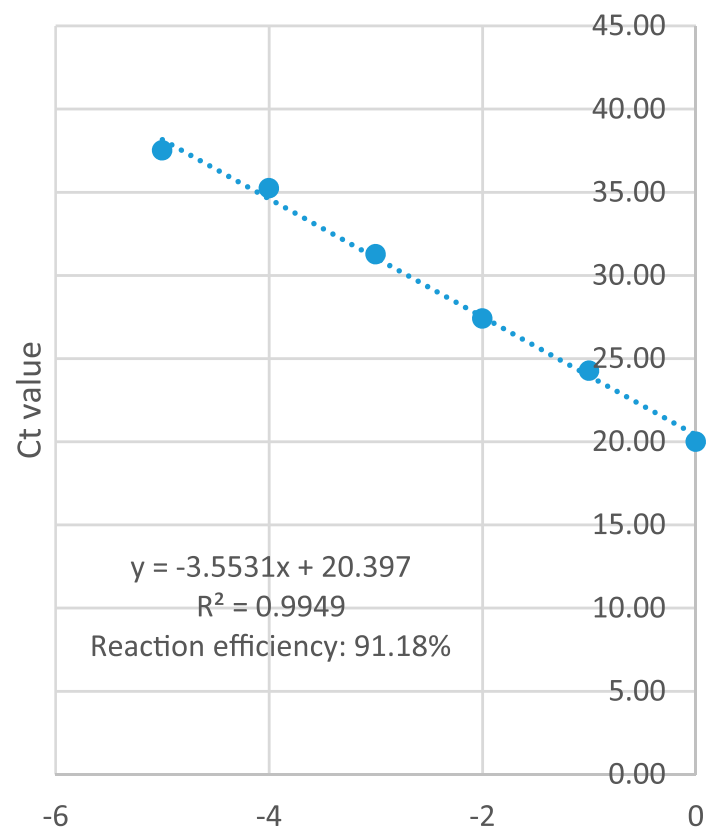

Log Concentration (ng)

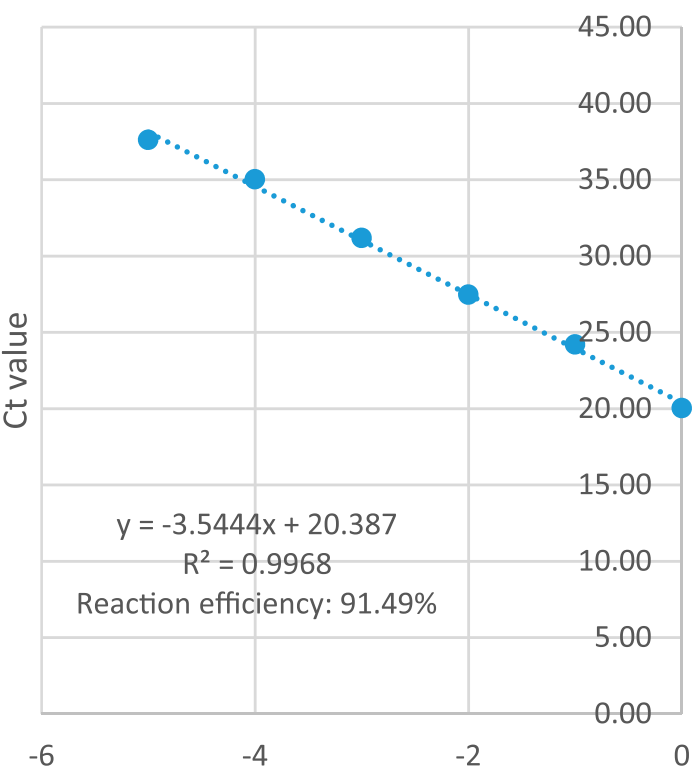

Log Concentration (ng)
B Sensitivity Assay of Fv-R

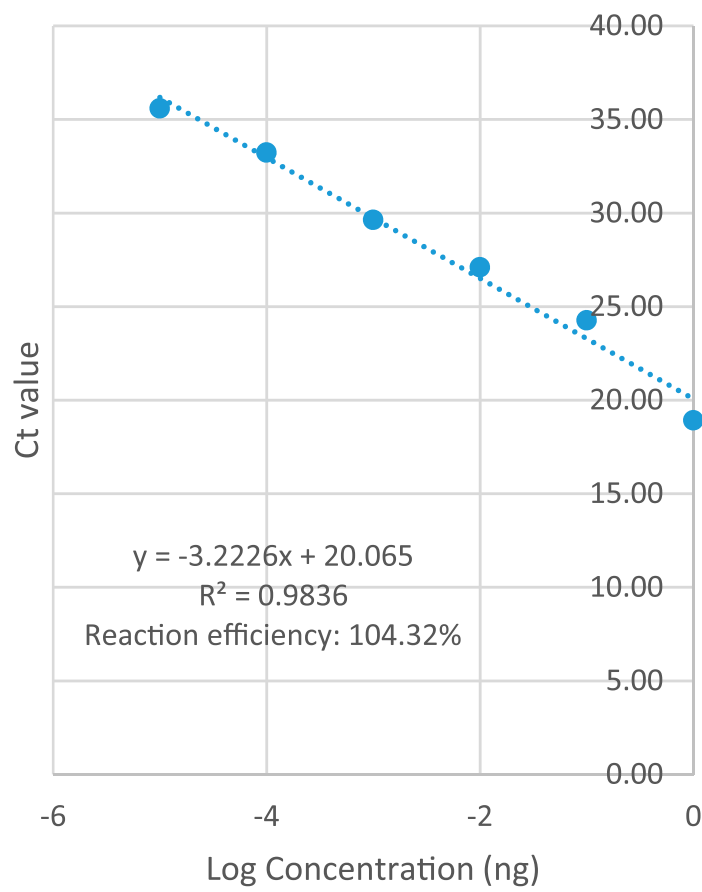

D Sensitivity Assay of Fv-R mixed with Fp-G DNA

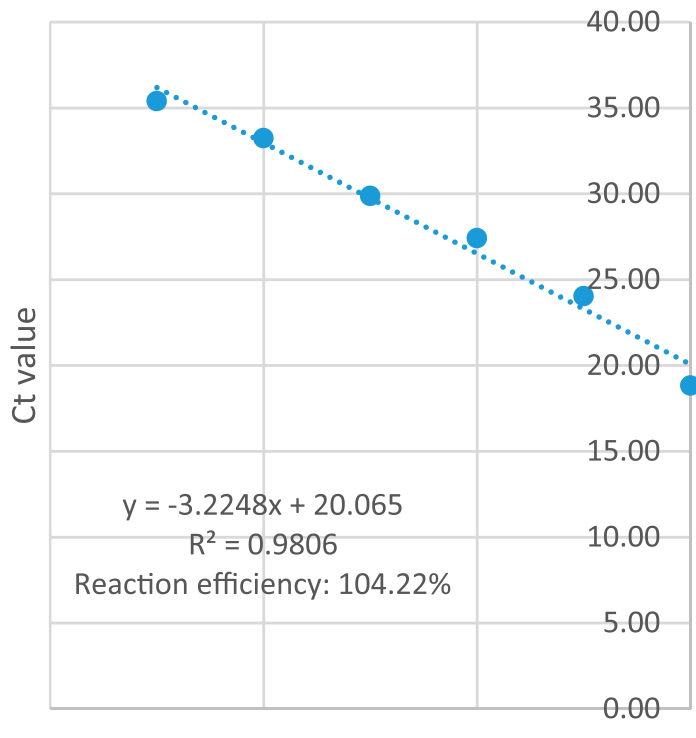

$-6$
$-2$

Log Concentration (ng)
0

(Continued)

Fig. 2. Standard curves were obtained for all real-time quantitative PCR. On the $x$-axis, DNA concentrations were logarithmically transformed to obtain a linear graph $(1 \mathrm{ng}=0,0.1$ $\mathrm{ng}=-1,0.01 \mathrm{ng}=-2,0.001 \mathrm{ng}=-3,0.0001 \mathrm{ng}=-4$, and $0.00001 \mathrm{ng}=-5$ ). Equations developed were used to calculate the amount of Fusarium proliferatum-green (Fp-G) and $F$. verticillioides-red $(F v-R)$ in the experiments. Reaction efficiency was calculated as described in the Bio-Rad real-time PCR applications guide using the formula percent efficiency $=$ $(E-1) \times 100 \%$, where $E=10^{(-1 / s l o p e)}$. Ct $=$ cycle threshold. A, Fp-G and B, Fv-R sensitivity assays; C, Fp-G and D, Fv-R sensitivity and specificity assays; $E$, Fp-G and F, Fv-R sensitivity and specificity inhibition assays spiked with maize root extract; and $\mathbf{G}, \mathrm{Fp}-\mathrm{G}$ and $\mathrm{H}$, Fv-R sensitivity and specificity inhibition assays spiked with maize stem extract. 
(Table 3). Treatments 2 and 9 were not different $(P=1)$ (Table 3). These results (comparison G) indicate that subsequent colonization by $F$. verticillioides (treatment 2 ) did not significantly impact $F$. proliferatum colonization of that plant (treatment 9 ). Also, interestingly, colonization by $F$. proliferatum-green in treatment 3 (cured plants) was not significantly different from that in treatments $1(F$. verticillioides-red-colonized plants; comparison $\mathrm{B}$ ) and treatment 5 (natural microflora; comparison $\mathrm{H}$ ). The latter data suggest that the presence of $F$. verticillioides (treatment 1 ) or of naturally occurring seedborne microorganisms (treatment 5 ) in the plant did not inhibit

Fig. 2. (Continued from previous page)

\section{E Spiked Assay of Fp-G and root extract}

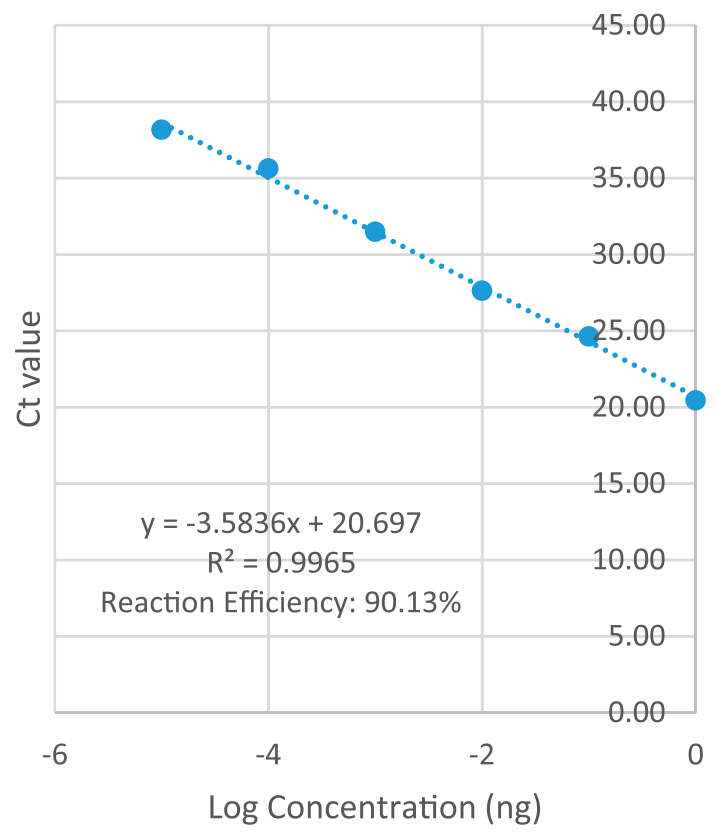

\section{G Spiked Assay of Fp-G and stem extract}

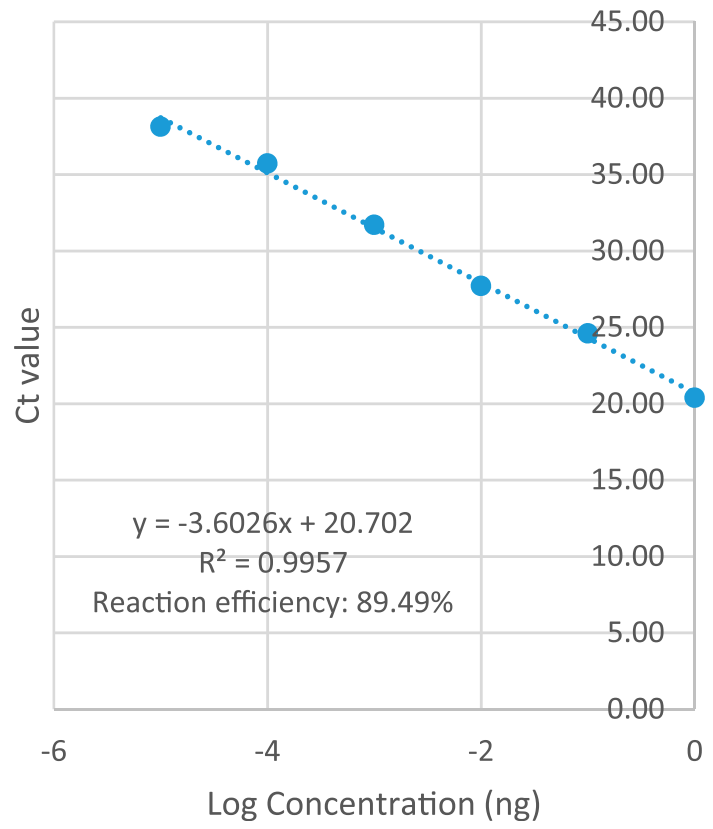

$F$. proliferatum from colonizing the maize plants from sources of inoculum in soil.

Colonization of roots by $F$. proliferatum-green $(7.38 \mathrm{pg})$ was 2.63 fold greater than colonization of stems by $F$. proliferatum-green $(2.80 \mathrm{pg})(P<0.0001)$ among treatments. An inverse gradient in the degree of colonization (fungal biomass) of the plant tissues as a function of the distance from the seed occurred $(P<0.0001)$; the most fungal biomass was found in the root and stem segments closest to the seed. The amount of $F$. proliferatum-green biomass in the segment closest to the seed (root and stem segment 1) was $2.91 \mathrm{pg}$, in the

\section{F Spiked Assay of Fv-R and root extract}

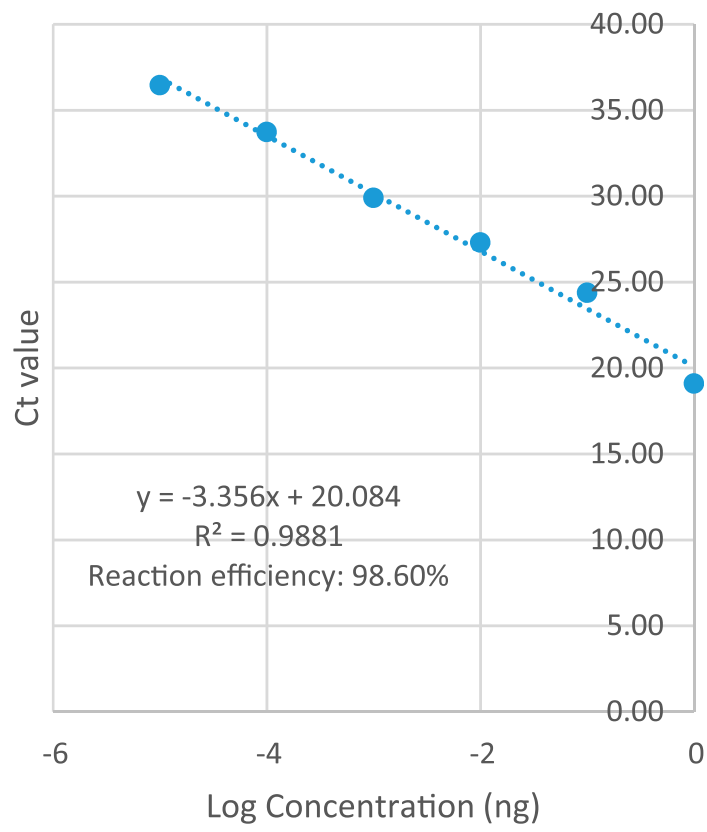

\section{H Spiked Assay of Fv-R and stem extract}

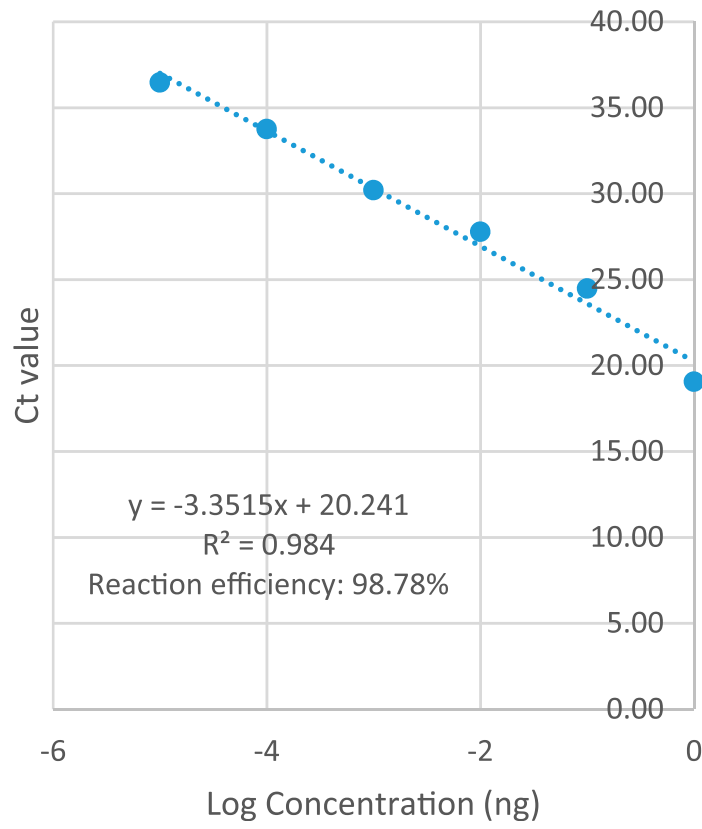


second segment closest to the seed (root and stem segment 2) was $1.78 \mathrm{pg}$, and in the segment farthest away from the seed (root and stem segment 3) was $0.40 \mathrm{pg}$. There were significant differences when comparing the $F$. proliferatum-green biomass in segment 1 to segment 2 , segment 1 to segment 3 , and segment 2 to segment 3 ( $P<0.0001$ for all comparisons).

The real-time qPCR data for $F$. verticillioides indicated that colonization of maize plants from sources of inoculum were different from colonization of maize plants from the inoculated seed $(P<$ 0.05 ) (Table 4). This was shown by the amount of $F$. verticillioides-red biomass detected in the plant tissues among the treatments (values shown in parenthesis in the comparisons). There were no significant differences among treatments $2(0.92 \mathrm{pg}), 4(1.79 \mathrm{pg})$, and $6(0.39 \mathrm{pg})$; however, these three treatments were significantly different from treatments $1(3.50 \mathrm{pg})$ and $8(3.39 \mathrm{pg})(P<0.05)$ (Table 4$)$. Treatments 1 and 8 were not different $(P=1)$ (Table 4$)$. These results (comparison $\mathrm{N}$ ) indicate that subsequent colonization by $F$. proliferatum (treatment 1 ) did not impact $F$. verticillioides colonization of that plant (treatment 8 ). Also, interestingly, colonization by $F$. verticillioides-red in treatment 4 (cured plants) did not differ from that in treatments $2(F$. proliferatum-green-colonized; comparison $\mathrm{O})$ and 6 (natural microflora; comparison R). The latter data suggest that the presence of $F$. proliferatum (treatment 2 ) or of naturally occurring seedborne microorganisms (treatment 6 ) in the plant did not inhibit $F$. verticillioides from colonizing maize plants from sources of inoculum in soil.

Colonization of roots by $F$. verticillioides-red $(4.88 \mathrm{pg}$ ) was $2.72-$ fold greater than colonization of the stems by $F$. verticillioides-red $(1.79 \mathrm{pg})(P<0.0001)$ among treatments. An inverse gradient in the degree of colonization (fungal biomass) of the plant tissues as a function of the distance from the seed occurred $(P<0.0001)$; the most fungal biomass was found in the root and stem segments closest to the seed. The amount of $F$. verticillioides-red biomass in the segment closest to the seed (root and stem segment 1) was $2.00 \mathrm{pg}$, in the second segment closest to the seed (root and stem segment 2) was $0.85 \mathrm{pg}$, and in the segment farthest away from the seed (root and stem segment 3) was $0.48 \mathrm{pg}$. There were significant differences when comparing the $F$. verticillioides-red biomass in segment 1 to segment 2, segment 1 to segment 3 , and segment 2 to segment 3 ( $P<0.0001$ for all comparisons).

\section{Discussion}

Biological invasions are the consequence of discrete events, including the passive or active introduction of an organism into a new environment, its subsequent establishment in the new environment, and, ultimately, an expansion of the area colonized. Current global seed production and distribution systems have established the pathways and mechanisms that facilitate the passive movement of plant pathogens across countries and continents where they are regularly, and repeatedly, introduced into new environments. $F$. proliferatum is seedborne and symptomless in many plant species, including maize (Logrieco et al. 2002; Munkvold 2003). Perhaps more important than its pathogenicity is its toxicity; $F$. proliferatum produces several toxins, the most important of which is the broadspectrum mycotoxin fumonisin (Leslie and Summerell 2006). Strains and populations of $F$. proliferatum vary widely in the amounts of toxin synthesized, sometimes by as much as four orders of magnitude. Cryptic movement of highly virulent or highly toxigenic strains in seed is a potential outcome.

This investigation demonstrated that $F$. proliferatum can grow from a source of inoculum in soil, infect a maize plant, and effectively compete against $F$. verticillioides and the natural maize

Table 3. Competitive colonization of maize plants by Fusarium proliferatum strain Fp-G in treatments 1, 2, 3, 5, and $9^{\text {a }}$

\begin{tabular}{|c|c|c|c|c|c|c|c|c|c|c|}
\hline Comp & Trt & Fp-G (pg) & Trt & Fp-G (pg) & Estimate & SE & DF & $t$ Value & $\operatorname{Pr}>|t|$ & Adjusted $P$ value ${ }^{b}$ \\
\hline $\mathrm{A}^{\mathrm{c}}$ & 1 & 1.28 & 2 & 5.39 & -2.7813 & 0.3839 & 8 & -7.24 & $<0.0001$ & 0.0009 \\
\hline $\mathrm{B}^{\mathrm{d}}$ & 1 & 1.28 & 3 & 2.22 & -0.8115 & 0.3839 & 8 & -2.11 & 0.0675 & 0.6749 \\
\hline $\mathrm{C}^{\mathrm{e}}$ & 1 & 1.28 & 5 & 0.94 & 0.3120 & 0.3839 & 8 & 0.81 & 0.4399 & 1.0000 \\
\hline$D^{f}$ & 1 & 1.28 & 9 & 5.45 & -2.6279 & 0.3839 & 8 & -6.84 & 0.0001 & 0.0013 \\
\hline$E^{g}$ & 2 & 5.39 & 3 & 2.22 & 1.9699 & 0.3839 & 8 & 5.13 & 0.0009 & 0.0089 \\
\hline$F^{h}$ & 2 & 5.39 & 5 & 0.94 & 3.0934 & 0.3839 & 8 & 8.06 & $<0.0001$ & 0.0004 \\
\hline $\mathrm{G}^{\mathrm{i}}$ & 2 & 5.39 & 9 & 5.45 & 0.1535 & 0.3839 & 8 & 0.40 & 0.6998 & 1.0000 \\
\hline $\mathrm{H}^{\mathrm{j}}$ & 3 & 2.22 & 5 & 0.94 & 1.1235 & 0.3839 & 8 & 2.93 & 0.0191 & 0.1910 \\
\hline $\mathrm{I}^{\mathrm{k}}$ & 3 & 2.22 & 9 & 5.45 & -1.8164 & 0.3839 & 8 & -4.73 & 0.0015 & 0.0148 \\
\hline $\mathrm{J}^{1}$ & 5 & 0.94 & 9 & 5.45 & -2.9399 & 0.3839 & 8 & -7.66 & $<0.0001$ & 0.0006 \\
\hline
\end{tabular}

a Colonization of maize plants from sources of inoculum (treatments 1, 3, and 5) showed significant differences from the colonization of maize plants from the inoculated seed (treatments 2 and 9). Colonization in treatment 3 (cured plants) was not significantly different from that in treatments 1 (comparison B) and 5 (comparison H). Comparisons (Comp) were done using the amount of Fp-G biomass, which was calculated from the cycle threshold values detected by real-time quantitative PCR. Quantification data were logarithmically transformed, and analysis was done using the GLIMMIX procedure (PROC GLIMMIX) in SAS Bonferroni correction of $P$ values were calculated to reduce chances of obtaining false-positive results due to the amount of comparisons performed. Trt $=$ treatment number and $\mathrm{SE}=$ standard error.

b $P$ values were adjusted with a Bonferroni correction.

c A compares the amount of Fp-G in maize plants in treatments 1 and 2. In treatment 1 , Fp-G was the source of inoculum and, in treatment 2, Fp-G was inoculated in the planted seed. In both treatments, Fp-G competed with $F$. verticillioides-red (Fv-R).

${ }^{\mathrm{d}} \mathrm{B}$ compares the amount of Fp-G in maize plants in treatments 1 and 3 . In both treatments, Fp-G was the source of inoculum. In treatment 1 , Fp-G competed with Fv-R and, in treatment 3, Fp-G did not have competition.

e C compares the amount of Fp-G in maize plants in treatments 1 and 5. In both treatments, Fp-G was the source of inoculum. In treatment 1 , Fp-G competed with Fv-R and, in treatment 5, Fp-G competed with natural seed microflora.

f $\mathrm{D}$ compares the amount of Fp-G in maize plants in treatments 1 and 9 . In treatment 1 , Fp-G was the source of inoculum and competed with Fv-R and, in treatment 9, Fp-G was inoculated in the planted seed and did not have competition.

g E compares the amount of Fp-G in maize plants in treatments 2 and 3. In treatment 2, Fp-G was inoculated in the planted seed and competed with Fv-R and, in treatment 3, Fp-G was the source of inoculum and did not have competition.

${ }^{\mathrm{h}} \mathrm{F}$ compares the amount of Fp-G in maize plants in treatments 2 and 5. In treatment 2, Fp-G was inoculated in the planted seed and competed with Fv-R and, in treatment 5, Fp-G was the source of inoculum and competed with natural seed microflora.

i $\mathrm{G}$ compares the amount of Fp-G in maize plants in treatments 2 and 9 . In both treatments, Fp-G was inoculated in the planted seed. In treatment 2 , Fp-G competed with Fv-R and, in treatment 9 , Fp-G did not have competition.

j $\mathrm{H}$ compares the amount of Fp-G in maize plants in treatments 3 and 5. In both treatments, Fp-G was the source of inoculum. In treatment 3 , Fp-G did not have competition and, in treatment 5, Fp-G competed with natural seed microflora.

${ }^{k}$ I compares the amount of Fp-G in maize plants in treatments 3 and 9. In treatment 3, Fp-G was the source of inoculum and, in treatment 9, Fp-G was inoculated in the planted seed. In both treatments, Fp-G did not have competition.

$1 \mathrm{~J}$ compares the amount of Fp-G in maize plants in treatments 5 and 9 . In treatment 5 , Fp-G was the source of inoculum and competed with natural seed microflora and, in treatment 9, Fp-G was inoculated in the planted seed and did not have competition. 
microflora for a niche in the roots and stems of developing maize plants. This research studied the infection and colonization of maize at the same time from either seedborne or soilborne inoculum sources (or both) from these two species. We used a TaqMan multiplex realtime qPCR approach with two primer and probe sets that were highly sensitive and specific to detect GFP for $F$. proliferatum and mRFP for $F$. verticillioides for quantification in maize plants. Southern blot analysis indicated a single insertion of GFP in the $F$. proliferatum-green genome and a single insertion of mRFP in the $F$. verticillioides-red genome; hence, the real-time qPCR data did not require transformation for analysis. Sensitivity and specificity of the primer and probes were important to obtain accurate and reliable results of maize plants colonized by $F$. proliferatum-green and $F$. verticillioides-red. No nonspecific reactions or crossreactions were observed for the two primer and probe sets. In addition, we found that the presence of maize tissue suspension did not inhibit or decrease the detection of $F$. proliferatum-green or $F$. verticillioides-red.

$F$. proliferatum can actively grow from a source of inoculum and colonize available noncolonized organic matter in a nonsterile soil under different temperature and soil matric potential combinations; this was true in a sieved, reconstituted field soil as well as in nonsterile soil with an intact field structure (Reyes Gaige et al. 2019). This investigation complements those findings by demonstrating that $F$. proliferatum and $F$. verticillioides can also colonize living plants already colonized with other microorganisms, even those likely to compete for the same substrate or niche. These Fusarium spp. were selected to compete against each other because of their ecological and life cycle similarity and common coexistence in maize plants. These two fungi systemically colonize maize plants, causing disease in different plant tissues and producing mycotoxins in kernels (Chulze et al. 2000; Leslie and Summerell 2006).

$F$. proliferatum can survive in soil for extended periods of time (Leslie et al. 1990; Logrieco and Bottalico 1988) and for at least 630 days in surface or buried crop residue (Cotten and Munkvold 1998). Similarly, F. verticillioides can survive for at least 630 days in surface or buried crop residue (Cotten and Munkvold 1998; Oren et al. 2003); however, this inoculum source has not been considered a major source of plant infection (Leslie et al. 1990; Munkvold and Carlton 1997).

$F$. proliferatum has worldwide distribution and a wide host range that includes both monocots and dicots, which increases the risk of infection of plants in a field because there may be multiple sources of inoculum, including soil. Genetic research on $F$. proliferatum indicates that strains vary in aggressiveness in certain crops (Elmer 1991; Iglesias et al. 2010) and that some strains can be predominant in some fields (Elmer 1991; Elmer et al. 1999). Moreover, there is genetic and phenotypic variation among strains from different plant hosts (Stępień et al. 2011), although host specialization has not been reported. Therefore, the introduction of $F$. proliferatum on one plant species may be an important source for infection or colonization of other plant species. This can occur where crop rotations of different plant species such as maize with wheat, soybean, or alfalfa are practiced. In addition, because the presence of $F$. proliferatum in plant and seed can be asymptomatic, this fungus can be inadvertently introduced into new environments, thereby extending the geographical range of undesirable strains.

In interspecific competition between fungi, environmental factors play an important role and influence the dominance of species (Magan and Lacey 1985). F. proliferatum and $F$. verticillioides have

Table 4. Competitive colonization of maize plants by Fusarium verticillioides strain $\mathrm{Fv}-\mathrm{R}$ in treatments 1, 2, 4, 6, and $8^{\mathrm{a}}$

\begin{tabular}{lcccccccccr}
\hline Comp & Trt & Fv-R $(\mathbf{p g})$ & Trt & Fv-R $(\mathbf{p g})$ & Estimate & SE & DF & $\boldsymbol{t}$ Value & Pr $>|\boldsymbol{t}|$ & Adjusted $\boldsymbol{P}$ value \\
\hline $\mathrm{K}^{\mathrm{c}}$ & 1 & 3.50 & 2 & 0.92 & 2.7999 & 0.3889 & 8 & 7.20 & $<0.0001$ \\
$\mathrm{~L}^{\mathrm{d}}$ & 1 & 3.50 & 4 & 1.80 & 1.9881 & 0.3889 & 8 & 5.11 & 0.0009 \\
$\mathrm{M}^{\mathrm{e}}$ & 1 & 3.50 & 6 & 0.39 & 3.1972 & 0.3889 & 8 & 8.22 & $<0.0001$ \\
$\mathrm{~N}^{\mathrm{f}}$ & 1 & 3.50 & 8 & 3.39 & 0.1357 & 0.3889 & 8 & 0.35 & 0.7362 & 0.0009 \\
$\mathrm{O}^{\mathrm{g}}$ & 2 & 0.92 & 4 & 1.80 & -0.8117 & 0.3889 & 8 & -2.09 & 0.0703 & 0.0004 \\
$\mathrm{P}$ & 2 & 0.92 & 6 & 0.39 & 0.3974 & 0.3889 & 8 & 1.02 & 0.3368 & 0.7032 \\
$\mathrm{Q}^{\mathrm{i}}$ & 2 & 0.92 & 8 & 3.39 & -2.6642 & 0.3889 & 8 & -6.85 & 0.0001 \\
$\mathrm{R}^{\mathrm{j}}$ & 4 & 1.80 & 6 & 0.39 & 1.2091 & 0.3889 & 8 & 3.11 & 0.0145 & 0.0000 \\
$\mathrm{~S}^{\mathrm{k}}$ & 4 & 1.80 & 8 & 3.39 & -1.8525 & 0.3889 & 8 & -4.76 & 0.0014 & 0.1447 \\
$\mathrm{~T}^{\mathrm{l}}$ & 6 & 0.39 & 8 & 3.39 & -3.0616 & 0.3889 & 8 & -7.87 & $<0.0001$ & 0.0142 \\
\hline
\end{tabular}

a Colonization of maize plants from sources of inoculum (treatments 2,4 , and 6 ) showed significant differences from the colonization of maize plants from the inoculated seed (treatments 1 and 8). Colonization in treatment 4 (cured plants) was not significantly different from that in treatments 2 (comparison $\mathrm{O}$ ) and 6 (comparison R). Comparisons (Comp) were done using the amount of Fv-R biomass, which was calculated from the cycle threshold values detected by real-time quantitative PCR. Quantification data were logarithmically transformed, and analysis was done using the GLIMMIX procedure (PROC GLIMMIX) in SAS Bonferroni correction of $P$ values were calculated to reduce chances of obtaining false-positive results due to the amount of comparisons performed. Trt $=$ treatment number and $\mathrm{SE}=$ standard error.

b $P$ values were adjusted with a Bonferroni correction.

${ }^{c} \mathrm{~K}$ compares the amount of FV-R in maize plants in treatments 1 and 2 . In treatment $1, \mathrm{Fv}-\mathrm{R}$ was inoculated in the planted seed and, in treatment 2 , Fv- $\mathrm{R}$ was the source of inoculum. In both treatments, Fv-R competed with F. proliferatum-green (Fp-G).

${ }^{\mathrm{d}} \mathrm{L}$ compares the amount of Fv-R in maize plants in treatments 1 and 4. In treatment 1, Fv-R was inoculated in the planted seed and competed with Fp-G and, in treatment $4, \mathrm{Fv}-\mathrm{R}$ was the source of inoculum and did not have competition.

e $\mathrm{M}$ compares the amount of Fv-R in maize plants in treatments 1 and 6 . In treatment 1 , Fv-R was inoculated in the planted seed and competed with Fp-G and, in treatment 6, Fv-R was the source of inoculum and competed with natural seed microflora.

${ }^{\mathrm{f}} \mathrm{N}$ compares the amount of Fv-R in maize plants in treatments 1 and 8 . In both treatments, Fv-R was inoculated in the planted seed. In treatment 1 , Fv-R competed with Fp-G and in treatment $8, \mathrm{Fv}-\mathrm{R}$ did not have competition.

g O compares the amount of Fv-R in maize plants in treatments 2 and 4 . In both treatments, Fv-R was the source of inoculum. In treatment 2 , Fv-R competed with Fp-G and, in treatment 4, Fv-R did not have competition.

h $\mathrm{P}$ compares the amount of Fv-R in maize plants in treatments 2 and 6 . In both treatments, Fv- $\mathrm{R}$ was the source of inoculum. In treatment 2 , Fv-R competed with Fp-G and, in treatment 6, Fv-R competed with natural seed microflora.

i Q compares the amount of Fv-R in maize plants in treatments 2 and 8 . In treatment 2, Fv-R was the source of inoculum and competed with Fp-G and, in treatment $8, \mathrm{Fv}-\mathrm{R}$ was inoculated in the planted seed and did not have competition.

j $\mathrm{R}$ compares the amount of FV-R in maize plants in treatments 4 and 6 . In both treatments, Fv- $\mathrm{R}$ was the source of inoculum. In treatment 4 , Fv- $\mathrm{R}$ did not have competition and, in treatment $6, \mathrm{Fv}-\mathrm{R}$ competed with natural seed microflora.

${ }^{k} \mathrm{~S}$ compares the amount of Fv-R in maize plants in treatments 4 and 8 . In treatment $4, \mathrm{FV}-\mathrm{R}$ was the source of inoculum and, in treatment 8 , Fv-R was inoculated in the planted seed. In both treatments, Fv-R did not have competition.

${ }^{1} \mathrm{~T}$ compares the amount of Fv-R in maize plants in treatments 6 and 8 . In treatment $6, \mathrm{Fv}-\mathrm{R}$ was the source of inoculum and competed with natural seed microflora and, in treatment $8, \mathrm{Fv}-\mathrm{R}$ was inoculated in the planted seed and did not have competition. 
similar abiotic requirements for optimal growth (Marín et al. 1996) and commonly occur together in plants, which makes them ideal to study competition. Understanding the competitive ability of these Fusarium spp. is essential to assessing the risk of entry and establishment of new strains into new environments via colonized seed.

\section{Acknowledgments}

We thank T. Fischer, M. Giraldo, and B. Valent for assistance with the Agrobacterium tumefaciens transformations; $\mathrm{M}$. Arif for assistance in designing the primers and probe sets used in this study; and E. Oliveira-Garcia and B. Valent for assistance with the Southern blot.

\section{Literature Cited}

Abendroth, L. J., Elmore, R. W., Boyer, M. J., and Marlay, S. K. 2011. Corn Growth and Development. PMR 1009. Iowa State University Extension, Ames, IA, U.S.A.

Arif, M., Fletcher, J., Marek, S. M., Melcher, U., and Ochoa-Corona, F. M. 2013. Development of a rapid, sensitive, and field-deployable razor ex BioDetection system and quantitative PCR assay for detection of Phymatotrichopsis omnivora using multiple gene targets. Appl. Environ. Microbiol. 79:2312-2320.

Chulze, S. N., Ramirez, M. L., Torres, A., and Leslie, J. F. 2000. Genetic variation in Fusarium section Liseola from no-till maize in Argentina. Appl. Environ. Microbiol. 66:5312-5315.

Cotten, T., and Munkvold, G. 1998. Survival of Fusarium moniliforme, F. proliferatum, and $F$. subglutinans in maize stalk residue. Phytopathology 88:550-555.

Daniels, B. 1983. Elimination of Fusarium moniliforme from corn seed. Plant Dis. 67:609-611.

Desjardins, A. E. 2006. Fusarium Mycotoxins: Chemistry, Genetics, and Biology. American Phytopathological Society Press, St. Paul, MN, U.S.A.

Desprez-Loustau, M.-L., Robin, C., Buée, M., Courtecuisse, R., Garbaye, J., Suffert, F., Sache, I., and Rizzo, D. M. 2007. The fungal dimension of biological invasions. Trends Ecol. Evol. 22:472-480.

Elmer, W. H. 1991. Vegetative compatibility groups of Fusarium proliferatum from asparagus and comparisons of virulence, growth rates, and colonization of asparagus residues among groups. Phytopathology 81:852-857.

Elmer, W. H. 2001. Seeds as vehicles for pathogen importation. Biol. Invasions 3: 263-271

Elmer, W. H., Summerell, B. A., Burgess, L. W., and Nigh, E. L. 1999. Vegetative compatibility groups in Fusarium proliferatum from asparagus in Australia. Mycologia 91:650-654.

Iglesias, J., Presello, D. A., Botta, G., Lori, G. A., and Fauguel, C. M. 2010. Aggressiveness of Fusarium Section Liseola isolates causing maize ear rot in Argentina. J. Plant Pathol. 92:205-211.

Leslie, J. F., Pearson, C. A., Nelson, P. E., and Toussoun, T. 1990. Fusarium spp. from corn, sorghum, and soybean fields in the central and eastern United States. Phytopathology 80:343-350.

Leslie, J. F., and Summerell, B. A., eds. 2006. The Fusarium Laboratory Manual. Blackwell Publishing, Ames, IA, U.S.A.

Leslie, J. F., Zeller, K. A., Logrieco, A., Mule, G., Moretti, A., and Ritieni, A. 2004. Species diversity of and toxin production by Gibberella fujikuroi species complex strains isolated from native prairie grasses in Kansas. Appl. Environ. Microbiol. 70:2254-2262.

Logrieco, A., and Bottalico, A. 1988. Fusarium species of the Liseola section associated with stalk and ear rot of maize in southern Italy, and their ability to produce moniliformin. Trans. Br. Mycol. Soc. 90:215-219.

Logrieco, A., Doko, B., Moretti, A., Frisullo, S., and Visconti, A. 1998. Occurrence of fumonisin B1 and B2 in Fusarium proliferatum infected asparagus plants. J. Agric. Food Chem. 46:5201-5204.

Logrieco, A., Mule, G., Moretti, A., and Bottalico, A. 2002. Toxigenic Fusarium species and mycotoxins associated with maize ear rot in Europe. Eur. J. Plant Pathol. 108:597-609.

Magan, N., and Lacey, J. 1984. Effect of water activity, temperature and substrate on interactions between field and storage fungi. Trans. Br. Mycol. Soc. 82:83-93.

Magan, N., and Lacey, J. 1985. Interactions between field, and storage fungi on wheat grain. Trans. Br. Mycol. Soc. 85:29-37.

Marasas, W. F. 1995. Fumonisins: Their implications for human and animal health. Nat. Toxins 3:193-198.

Marasas, W. F., Riley, R. T., Hendricks, K. A., Stevens, V. L., Sadler, T. W., Gelineau-van Waes, J., Missmer, S. A., Cabrera, J., Torres, O., Gelderblom, W. C., and Allegood, J. 2004. Fumonisins disrupt sphingolipid metabolism, folate transport, and neural tube development in embryo culture and in vivo: A potential risk factor for human neural tube defects among populations consuming fumonisin-contaminated maize. J. Nutr. 134:711-716.

Marín, S., Sanchis, V., Arnau, F., Ramos, A., and Magan, N. 1998a. Colonisation and competitiveness of Aspergillus and Penicillium species on maize grain in the presence of Fusarium moniliforme and Fusarium proliferatum. Int. J. Food Microbiol. 45:107-117.

Marin, S., Sanchis, V., and Magan, N. 1995. Water activity, temperature, and pH effects on growth of Fusarium moniliforme and Fusarium proliferatum isolates from maize. Can. J. Microbiol. 41:1063-1070.

Marin, S., Sanchis, V., Ramos, A. J., Vinas, I., and Magan, N. 1998b. Environmental factors, in vitro interactions, and niche overlap between Fusarium moniliforme, F. proliferatum, and F. graminearum, Aspergillus and Penicillium species from maize grain. Mycol. Res. 102:831-837.

Marín, S., Sanchis, V., Rull, F., Ramos, A. J., and Magan, N. 1998c. Colonization of maize grain by Fusarium moniliforme and Fusarium proliferatum in the presence of competing fungi and their impact on fumonisin production. J. Food Prot. 61:1489-1496.

Marín, S., Sanchis, V., Teixido, A., Saenz, R., Ramos, A., Vinas, I., and Magan, N. 1996. Water and temperature relations and microconidial germination of Fusarium moniliforme and Fusarium proliferatum from maize. Can. J. Microbiol. 42:1045-1050.

Mullins, E., Chen, X., Romaine, P., Raina, R., Geiser, D., and Kang, S. 2001. Agrobacterium-mediated transformation of Fusarium oxysporum: An efficient tool for insertional mutagenesis and gene transfer. Phytopathology 91:173-180.

Munkvold, G. P. 2003. Epidemiology of Fusarium diseases and their mycotoxins in maize ears. Eur. J. Plant Pathol. 109:705-713.

Munkvold, G. P., and Carlton, W. M. 1997. Influence of inoculation method on systemic Fusarium moniliforme infection of maize plants grown from infected seeds. Plant Dis. 81:211-216.

O’Donnell, K., Cigelnik, E., and Nirenberg, H. I. 1998a. Molecular systematics and phylogeography of the Gibberella fujikuroi species complex. Mycologia 90:465-493.

O’Donnell, K., Kistler, H. C., Cigelnik, E., and Ploetz, R. C. 1998b. Multiple evolutionary origins of the fungus causing Panama disease of banana: Concordant evidence from nuclear and mitochondrial gene genealogies. Proc. Natl. Acad. Sci. U.S.A. 95:2044-2049.

Oren, L., Ezrati, S., Cohen, D., and Sharon, A. 2003. Early events in the Fusarium verticillioides-maize interaction characterized by using a green fluorescent protein-expressing transgenic isolate. Appl. Environ. Microbiol. 69:1695-1701

Pascale, M., Visconti, A., and Chelkowski, J. 2002. Ear rot susceptibility and mycotoxin contamination of maize hybrids inoculated with Fusarium species under field conditions. Eur. J. Plant Pathol. 108:645-651.

Ramakrishna, N., Lacey, J., and Smith, J. 1993. Effects of water activity and temperature on the growth of fungi interacting on barley grain. Mycol. Res. 97:1393-1402.

Reyes Gaige, A., Giraldo, M., Todd, T., and Stack, J. P. 2019. Growth and colonization of organic matter in soil by Fusarium proliferatum. Can. J. Plant Pathol. 41:242-250.

Reyes Gaige, A., and Stack, J. P. 2015. Interspecific competition for colonization of maize host between Fusarium proliferatum and Fusarium verticillioides. 2015 APS Annual Meeting. https://www.apsnet.org/meetings/Documents/ 2015_meeting_abstracts/aps2015abP475.htm

Riley, R. T., Wang, E., Schroeder, J. J., Smith, E. R., Plattner, R. D., Abbas, H., Yoo, H., and Merrill, A. H. 1996. Evidence for disruption of sphingolipid metabolism as a contributing factor in the toxicity and carcinogenicity of fumonisins. Nat. Toxins 4:3-15.

Ross, P. F., Nelson, P. E., Richard, J. L., Osweiler, G. D., Rice, L. G., Plattner, R. D. and Wilson, T. M. 1990. Production of fumonisins by Fusarium moniliforme and Fusarium proliferatum isolates associated with equine leukoencephalomalacia and a pulmonary edema syndrome in swine. Appl. Environ. Microbiol. 56:3225-3226

Stępien, Ł., Koczyk, G., and Waśkiewicz, A. 2011. Genetic and phenotypic variation of Fusarium proliferatum isolates from different host species. J. Appl. Genet. 52:487-496.

Thiel, P. G., Marasas, W. F., Sydenham, E. W., Shephard, G. S., and Gelderblom, W. C. 1992. The implications of naturally occurring levels of fumonisins in corn for human and animal health. Mycopathologia 117:3-9.

Verhoeven, K. J., Simonsen, K. L., and McIntyre, L. M. 2005. Implementing false discovery rate control: Increasing your power. Oikos 108:643-647.

Zuker, M. 2003. Mfold web server for nucleic acid folding and hybridization prediction. Nucleic Acids Res. 31:3406-3415. 